\title{
Beam-Ion Acceleration during Edge Localized Modes in the ASDEX Upgrade Tokamak
}

\author{
J. Galdon-Quiroga, ${ }^{1,2, *}$ M. Garcia-Munoz, ${ }^{1,2}$ K. G. McClements, ${ }^{3}$ M. Nocente, ${ }^{4}$ M. Hoelzl,${ }^{5}$ A. S. Jacobsen, ${ }^{5}$ \\ F. Orain, ${ }^{5}$ J. F. Rivero-Rodriguez, ${ }^{2,6}$ M. Salewski, ${ }^{7}$ L. Sanchis-Sanchez, ${ }^{1,2}$ W. Suttrop, ${ }^{5}$ E. Viezzer ${ }^{1,2}$ \\ the ASDEX Upgrade Team and the Eurofusion MST1 Team
}

\author{
${ }^{1}$ Department of Atomic, Molecular and Nuclear Physics, University of Seville, 41012 Seville, Spain \\ ${ }^{2}$ Centro Nacional de Aceleradores (CNA), Universidad de Sevilla, Junta de Andalucia, Consejo Superior de Investigaciones \\ Cientificas (CSIC), Parque Cientifico y Tecnologico Cartuja, c/Thomas Alva Edison, 741092 Seville, Spain \\ ${ }^{3}$ CCFE, Culham Science Centre, Abingdon OX14 3DB, United Kingdom \\ ${ }^{4}$ Dipartimento di Fisica G Occhialini, Universita di Milano-Bicocca, Milano, Italy \\ ${ }^{5}$ Max Planck Institute for Plasma Physics, Boltzmannstrasse, 285748 Garching bei Munchen, Germany \\ ${ }^{6}$ Department of Mechanical Engineering and Manufacturing, University of Seville, \\ Camino de los Descubrimientos s/n, 41092 Seville, Spain \\ ${ }^{7}$ Department of Physics, Technical University of Denmark, Kongens Lyngby, Denmark
}

(Received 11 January 2018; revised manuscript received 9 April 2018; published 10 July 2018)

\begin{abstract}
The acceleration of beam ions during edge localized modes (ELMs) in a tokamak is observed for the first time through direct measurements of fast-ion losses in low collisionality plasmas. The accelerated beamion population exhibits well-localized velocity-space structures which are revealed by means of tomographic inversion of the measurement, showing energy gains of the order of tens of keV. This suggests that the ion acceleration results from a resonant interaction between the beam ions and parallel electric fields arising during the ELM. Orbit simulations are carried out to identify the mode-particle resonances responsible for the energy gain in the particle phase space. The observation motivates the incorporation of a kinetic description of fast particles in ELM models and may contribute to a better understanding of the mechanisms responsible for particle acceleration, ubiquitous in astrophysical and space plasmas.
\end{abstract}

DOI: 10.1103/PhysRevLett.121.025002

The spontaneous acceleration of charged particles is ubiquitous in space, astrophysical, and laboratory plasmas [1-3]. Among these processes, magnetic reconnection appears often as a common agent in different environments such as solar flares [4] or Earth's magnetosphere [2] and magnetotail $[5,6]$. However, the identification of the acceleration mechanism is sometimes unclear, and, depending on the particular phenomena, different options are invoked [6-10]. In this sense, laboratory plasmas with in situ measurements may help to elucidate the underlying physics responsible for the acceleration mechanisms [3]. In the presence of an electric field, there is a certain threshold above which the accelerating force experienced by charged particles overcomes the drag force due to collisions, thus leading to the so-called runaway acceleration process [11]. In tokamaks, electron $[12,13]$ and ion $[14,15]$ runaways are

Published by the American Physical Society under the terms of the Creative Commons Attribution 4.0 International license. Further distribution of this work must maintain attribution to the author(s) and the published article's title, journal citation, and DOI. commonly observed in the presence of sub-Dreicer electric fields arising during internal magnetic reconnection. The acceleration of electrons during edge localized modes (ELMs) was reported recently [16], showing that such processes could play a role at the edge of tokamak plasmas. ELMs $[17,18]$ are periodic magnetohydrodynamic instabilities that appear in the edge-pedestal region of tokamak plasmas operating in the high confinement mode ( $H$ mode) [19]. These instabilities driven by an edge current and/or pressure gradient produce a cyclic loss of heat and particles to the walls which could limit the lifetime of plasma-facing components in future fusion reactors [20]. It is therefore of paramount importance to understand the physics underlying the interaction between ELMs and energetic particles.

In this Letter, we present, for the first time, evidence of beam-ion acceleration during ELMs in the ASDEX Upgrade (AUG) tokamak through direct measurements of fast-ion losses. The measurements have been performed in low pedestal collisionality plasmas $\left(\nu^{*} \leq 0.4\right)$ with $\beta_{N}=\beta\left[\left(a B_{t}\right) / I_{p}\right] \sim 2.5$, where $\beta$ is the ratio of plasma pressure to magnetic pressure, $a$ is the minor radius of the tokamak, $B_{t}$ is the toroidal magnetic field, and $I_{p}$ is the plasma current. In these experiments, the only external 

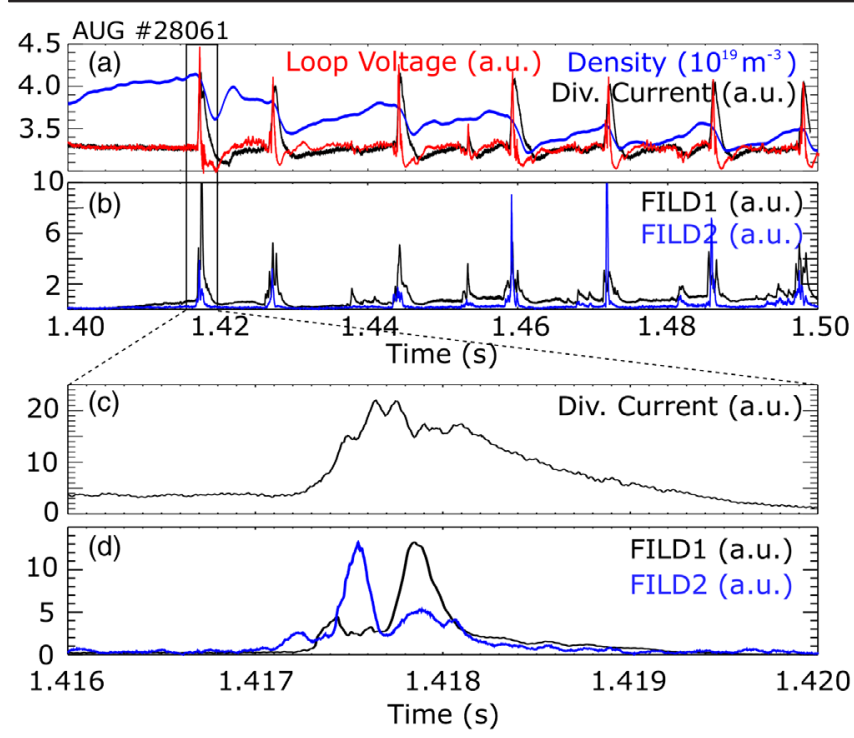

FIG. 1. (a) Time traces of the edge electron density (in blue), the loop voltage (in red), and the divertor current (in black). (b) Time traces of two different FILD signals. (c) and (d) show an enlargement of an individual ELM.

source of suprathermal ions to the plasma were high-energy neutral beam-injected (NBI) deuterium ions. Measurements with multiple scintillator-based fast-ion loss detectors (FILDs) [21], located near the AUG midplane at $z \sim$ $0.3 \mathrm{~m}$ and toroidally displaced $\Delta \Phi \sim 113^{\circ}$, show bursts of fast ions synchronized with the occurrence of ELM perturbations. In Fig. 1(a), the time trace of the divertor current (in black), which is used as an ELM monitor, and the line-integrated edge density (in blue), which is observed to drop at each ELM, are plotted. Spikes in the loop voltage signal (in red) are also observed during the ELMs, which are indicative of the appearance of transient electric fields. Figure 1(b) shows the time trace of the fast-ion losses measured by two different FILDs, which are located at the same poloidal position near the midplane but at different toroidal locations. An enlargement of an individual ELM is shown in Figs. 1(c) and 1(d). Filamentarylike behavior is observed in the FILD signals, where multiple spikes with characteristic times of $\sim 100 \mu \mathrm{s}$ occur within a single ELM crash, which has a longer characteristic time, typically on the order of $1 \mathrm{~ms}$. These fast-ion filaments are different for the two FILDs, thus revealing the 3D nature of the phenomenon. Detailed measurements of the velocity space of fast-ion losses in ELMs reveal a fast-ion population at energies well above the beam injection energy. This is shown in Fig. 2(a), where two main populations can be identified at pitch angles of $\arccos \left[-\left(v_{\|} / v\right)\right] \sim 45^{\circ}$ and $\sim 60^{\circ}$. These correspond to first orbit fast-ion losses of a tangential $(Q 7)$ and a radial $(Q 8)$ NBI source, identified as passing and trapped orbits, respectively, which explore the edge region of the plasma. Both populations show a similar pattern in the gyroradius profile: two spots centered
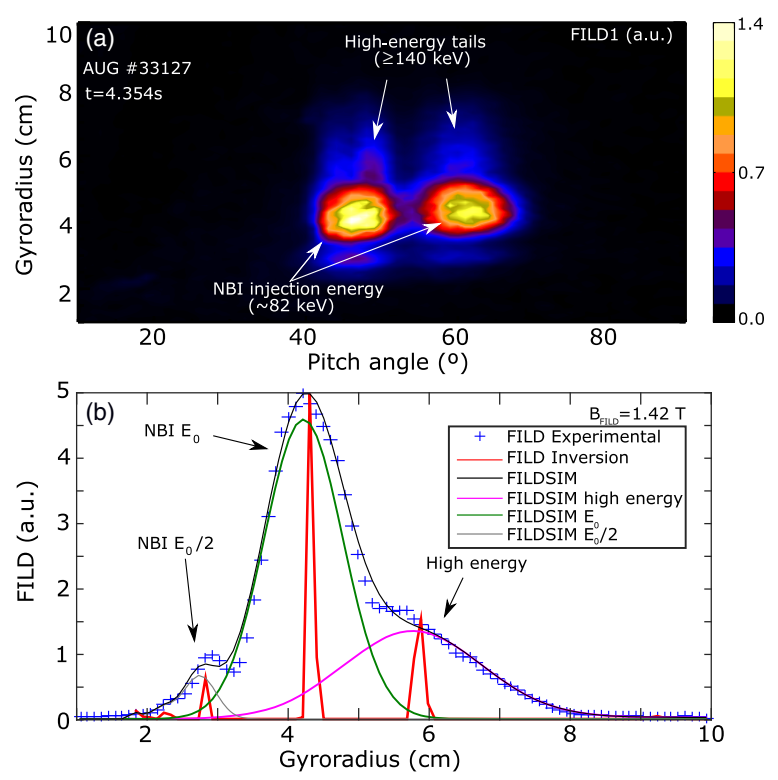

FIG. 2. (a) Velocity space of fast-ion losses measured by a FILD during an ELM. (b) Gyroradius profile of the FILD signal. The blue crosses indicate the experimental FILD signal. In red, the undistorted gyroradius profile obtained after the tomographic inversion is plotted. The black curve is the expected gyroradius profile for the inverted distribution.

at $r_{L} \sim 4 \mathrm{~cm}$ and $r_{L} \sim 5 \mathrm{~cm}$, which correspond to the main and half NBI energy components of $E_{0}=82 \mathrm{keV}$ and $\left(E_{0} / 2\right)=41 \mathrm{keV}$, respectively, and an additional population at $r_{L} \geq 5 \mathrm{~cm}$ which is observed only during the transient ELM crash and corresponds to energies well above the main NBI energy component, which shall be referred to as a high-energy feature onwards.

Because of the finite resolution of the FILD system, the velocity-space measurement at the scintillator plate is a distortion of the velocity space of the ions that reach the detector pinhole. The latter can be retrieved by applying tomographic inversion techniques to the measurements [22,23], using the FILDSIM model which takes into account the detector response [24,25]. Figure 2(b) shows the result of this analysis, where the gyroradius profile of the FILD signal is plotted, integrated along the pitch angle interval between $58^{\circ}$ and $65^{\circ}$. The blue crosses indicate the experimental measurement. The curve shows three bumps which are smooth due to the effect of the instrument resolution. After applying the velocity-space tomographic inversion to the experimental signal, we obtain the undistorted distribution, which is plotted in red. Three peaks which are very well localized in gyroradius are observed. The ones at $r_{L}=4.1 \mathrm{~cm}$ and $r_{L}=2.9 \mathrm{~cm}$ match perfectly with the main and half NBI energy components, respectively, which is expected for first orbit losses. Surprisingly, the high-energy feature is also very well localized in energy at a gyroradius of $r_{L}=5.8 \mathrm{~cm}$, which corresponds to $\sim 160 \mathrm{keV}$. The synthetic FILD signal expected for such 
a distribution is plotted in black, showing good agreement with the experimental measurement.

The observation of the high-energy feature is reproducible and has been observed over a wide range of parameters from $I_{p}=0.8-1 \mathrm{MA}$ and $B_{t}=1.8-2.5 \mathrm{~T}$, with $q_{95}$ varying from 3.5 to 5.5 approximately, thus including different toroidal mode numbers within the range expected in AUG. It is well correlated with the NBI heating systems and with the divertor current signal. This is illustrated in Fig. 3(a), showing the time evolution of the FILD gyroradius profile, together with the time traces of the divertor current (in white) and the NBI power (in red). Until $1.20 \mathrm{~s}$, no signal is measured in the FILD, in spite of an ELM occurring at $1.173 \mathrm{~s}$. At $1.20 \mathrm{~s}$, the NBI source delivering first orbit losses to the FILD is switched on, and the FILD starts to measure the main and half NBI energy components centered at $r_{L}=4.1 \mathrm{~cm}$ and $r_{L}=2.9 \mathrm{~cm}$. From this time point onwards, every time an ELM crash occurs, the intensity of the fast-ion losses at both NBI energy components is increased and the high-energy feature appears.

Further evidence of the correlation between the observed high-energy feature and the ELMs was found in an ELMsuppressed regime, achieved via the application of external magnetic perturbations (MPs) at AUG [26,27]. Figure 3(b) shows the evolution of the FILD gyroradius profile together with the divertor current time trace (in white) in such a case. As soon as the MPs are applied (indicated by the gray box), the ELMs are mitigated, their frequency is increased, and their amplitude is decreased. The high-energy feature is observed in both mitigated and unmitigated ELMs. At
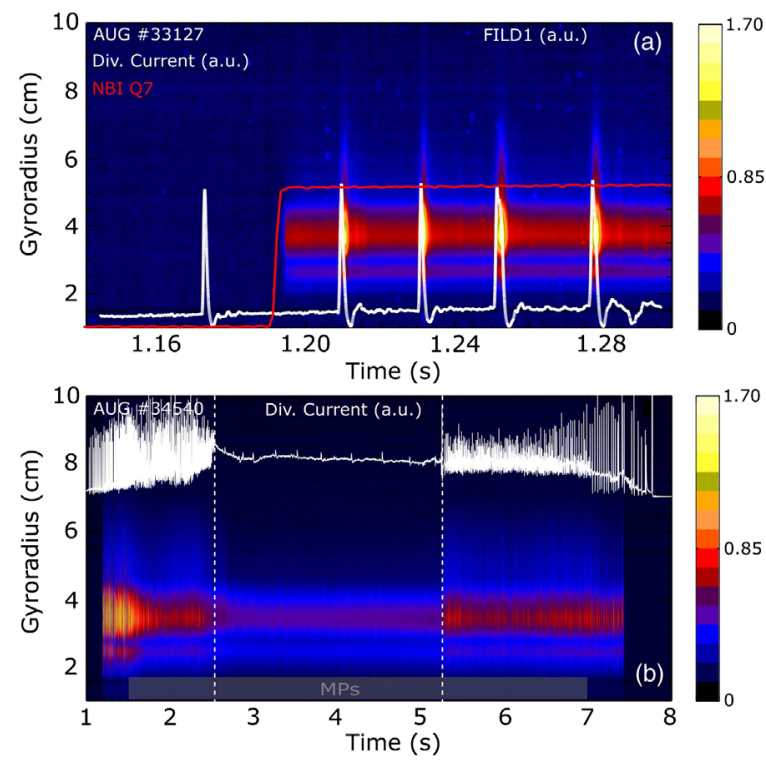

FIG. 3. Gyroradius profile evolution of the FILD signal in two AUG discharges. The divertor current is plotted in white. (a) Discharge no. 33127 without MPs. The NBI time trace is plotted in red. (b) Discharge no. 34540 with MPs (gray box). The time window in which ELM suppression is achieved is indicated by two vertical dashed lines. $t=2.5 \mathrm{~s}$, the ELM-suppressed regime is established and the high-energy feature disappears from the FILD signal, until $t=5.2 \mathrm{~s}$, when the ELM mitigated regime is recovered.

The high-energy feature shows a pitch angle structure that depends on the beam source and $q_{95}$, defined as the safety factor at $95 \%$ of the plasma minor radius. The pitch angle distribution of the main and half NBI energy components is not observed to vary during the ELM crashes. This is illustrated in Fig. 4(a), which shows the pitch angle profile of the FILD signal for the main NBI injection energy (in black, for reference) and for the highenergy feature component (in colors). The different colors correspond to different time points of a discharge in which a scan in $q_{95}$ was performed by ramping the toroidal magnetic field from 2.0 to $1.7 \mathrm{~T}$. The profile at the main NBI injection energy shows two smooth bumps centered at $45^{\circ}$ and $60^{\circ}$, corresponding to the first orbit losses of $Q 7$ and $Q 8$, respectively. For the pitch angle interval between $40^{\circ}$ and $50^{\circ}$ corresponding to source $Q 7$ (passing orbits), we observe that the high-energy component profile shows two different spikes centered approximately at $43^{\circ}$ (spike I) and $48^{\circ}$ (spike II). The relative intensity of the two spikes evolves as $q_{95}$ is ramped during the discharge. As $q_{95}$ decreases, the amplitude of spike I increases, while the amplitude of spike II decreases. For the pitch angle interval between $55^{\circ}$ and $65^{\circ}$, corresponding to source $Q 8$ (trapped orbits), no clear pitch angle evolution was observed.

The observation of the pitch angle structure in the highenergy feature, together with the results of the tomographic inversion, suggests a mechanism for the acceleration of the beam ions which is highly velocity-space dependent. Moreover, the characteristic times associated with the individual fast-ion filaments $(t \sim 100 \mu \mathrm{s})$ in comparison with the fast-ion slowing-down time in these plasmas
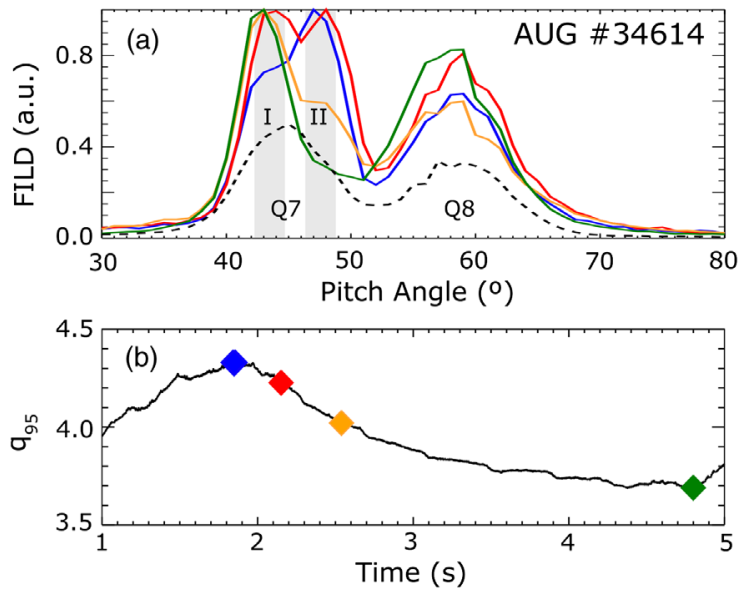

FIG. 4. (a) Pitch angle profile of the FILD signal. In black (dashed line), the profile at the main NBI injection energy. In color, the profile at the high-energy component for different time points indicated in (b), which shows the temporal evolution of $q_{95}$. 
$\left(\tau_{s} \sim 100 \mathrm{~ms}\right)$ indicate that the phenomenon is likely to be collisionless. While turbulent mechanisms are found to be relevant for particle transport and acceleration in space and astrophysical plasmas, the effect of turbulence on fast ions is thought to be negligible in these experiments provided the large $E / T_{e}$ ratio $(\geq 100)[28,29]$. We therefore propose a resonant interaction between the beam-ion orbits and the parallel electric field emerging during the ELM, when magnetic reconnection is believed to take place [18]. Only the ions whose orbits are in phase with the parallel electric field are subject to a net energy gain. Additionally, spikes in the soft $\mathrm{x}$-ray and bursts in the electron cyclotron emission signals have also been observed at the onset of some of these ELMs. These are indicative of electron acceleration [16], which further supports the hypothesis of the particle acceleration during ELMs in the ASDEX Upgrade tokamak.

The measured ELM induced beam-ion loss and acceleration are likely the result of a complex mechanism that includes the resonant interaction of the fast ions with the induced 3D electromagnetic perturbation. These phenomena have been modeled by performing full-orbit fastparticle following simulations in AUG realistic magnetic equilibria, including 3D magnetic and electric field perturbations, and in the absence of collisions with the background plasma. In order to investigate the ELMinduced beam-ion losses, these simulations have been carried out for a complete ELM crash including realistic magnetic field perturbations calculated with the 3D nonlinear resistive magnetohydrodynamics code JOREK [30]. Figure 5(a) shows how the magnetic perturbation associated with the ELM is located at the edge of the plasma. A
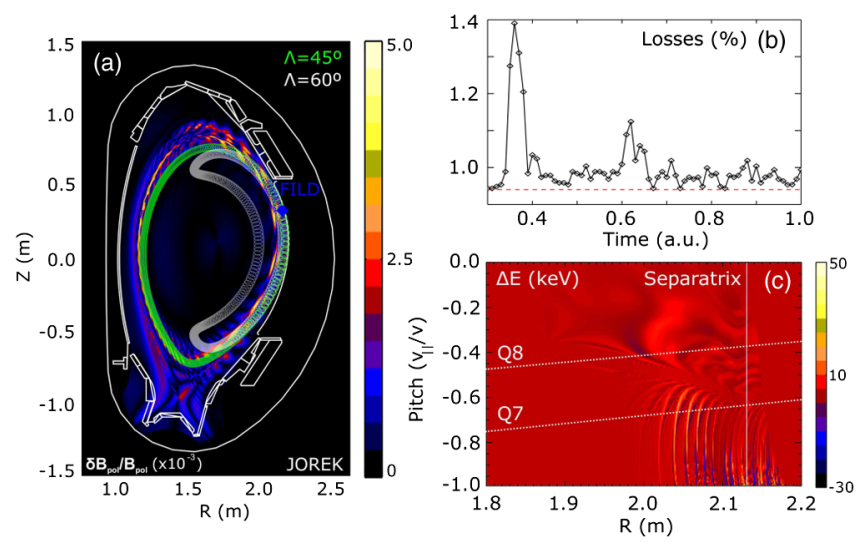

FIG. 5. (a) Poloidal contour of the magnetic perturbation of an ELM calculated with JOREK. Typical orbits measured by FILDs are overlaid. (b) Time trace of simulated fast-ion losses in a full ELM crash. The level of fast-ion losses without the perturbation is indicated for reference by a dashed red line. (c) Simulated energy gain of the markers in the presence of a parallel electric field given by Eq. (1). The separatrix position is indicated in white. The approximated deposition of beams $Q 7$ and $Q 8$ is indicated by the dotted white lines. clear poloidal overlap is observed with passing (green) and trapped (white) fast-ion orbits corresponding to those populations measured by the FILD. A realistic NBI birth distribution is calculated, and the markers are started at each of the different magnetic configurations throughout the whole ELM crash. The results of the simulation are shown in Fig. 5(b), where the intensity of the fast-ion losses integrated in the whole vessel is plotted. A number of spikes can be identified, reproducing the filamentarylike behavior observed in the FILD signals. The level of fast-ion losses in the absence of the perturbation is indicated by a dashed red line for reference.

While the temporal evolution of the losses is qualitatively reproduced using the JOREK 3D magnetic perturbation, an electric field is needed to account for the observed energy gain. In order to assess the viability of the proposed acceleration mechanism, simulations have been carried out in a realistic magnetic equilibrium including a simple test model for the parallel electric field, which is kept static in the simulation:

$$
\vec{E}=\overrightarrow{b_{\|}} \cdot A \exp \frac{\left(\rho-\rho_{0}\right)^{2}}{2 \sigma^{2}} \cos \left(n \phi-m \theta^{*}+\alpha\right),
$$

where $\overrightarrow{b_{\|}}$is a unitary vector parallel to the magnetic field, $A$ is a parameter controlling the amplitude of the electric field, $\rho_{0}$ and $\sigma$ are the centroid and width, respectively, of the perturbation in minor radius $\rho$, and $n$ and $m$ are the toroidal and poloidal mode numbers, respectively. $\phi$ is the toroidal angle coordinate, $\theta^{*}$ is the poloidal angle coordinate, and $\alpha$ is the phase of the perturbation. This simple parametrized model resembles the 3D character of the expected parallel electric field and allows us to perform a sensitivity scan in the main parameters of the perturbation (i.e., the amplitude of the parallel electric field and its spatial structure). A set of $10^{5}$ markers were initialized performing a scan in the radial coordinate $R$ and pitch $\Lambda=\left(v_{\|} / v\right)$ defined with respect to the magnetic field, with a fixed energy of $90 \mathrm{keV}$, corresponding to the main injection energy of AUG systems. The markers were followed during $50 \mu \mathrm{s}$, while the toroidal mode number was set to $n=10$, which is in the range of ELM observations in AUG [31], and the poloidal mode number was set as $m=q n$, where $q$ is the safety factor at $\rho_{0}$. The amplitude of the parallel electric field was set to $2 \mathrm{kV} / \mathrm{m}$, on the order of magnitude predicted by the modeling and well above the Dreicer electric field for these plasmas. The perturbation is centered in the pedestal edge region (i.e., $\rho_{\text {pol }}=0.9$ ) and an extension comparable to the pedestal width $(\sigma=0.1)$. Figure 5(c) shows the energy gain of the markers in the simulation. Only the particles exploring the edge region gain or lose energy. The maximum energy gain obtained is on the order of tens of $\mathrm{keV}$, which is in agreement with the experimental observation. Vertical structures are observed for the ions in the passing region $(\Lambda \leq-0.5)$, while not such a clear 
pattern is observed for the ions in the trapped region $(\Lambda \geq-0.5)$. Linear and nonlinear resonances between the particles and the perturbation [32] might be responsible for these structures, similar to recent observations in plasmas with externally applied 3D perturbative fields [33]. This is also in line with the experimental observation of the pitch angle structure dependence with $q_{95}$, which was found to be very clear for the passing orbits populated by beam source $Q 7$ but not for the trapped orbits of $Q 8$. By ramping $q_{95}$, the radial location being probed with the FILD is changing, and we move horizontally in Fig. 5(c). For orbits in the passing region, where the resonances are clear vertical structures, we would be jumping from one resonance to another. However, for orbits in the trapped region, where the structure of the resonances is more complicated, moving along the radial coordinate does not necessarily lead to a different resonance. In this framework, the change in the pitch angle structure measured with the FILD could then be understood as the probing of different resonances for different $q_{95}$ values. Additional simulations have been performed varying the $3 \mathrm{D}$ structure $\left(n, \rho_{0}, \sigma\right)$ and amplitude $(A)$ of the electric field, within the range of values expected for different ELMs. The results showed the same qualitative behavior for the resonant structures in the passing and trapped orbit regions and energy gains of the order of tens of $\mathrm{keV}$. Larger values of the amplitude of the parallel electric field, keeping the rest of the parameters constant, lead to an increase in the maximum energy gain as expected. On the other hand, the variation of the toroidal mode number shows that the maximum energy gain is larger for lower toroidal mode numbers.

In conclusion, the first evidence of beam-ion acceleration during ELMs has been presented through direct timeresolved velocity-space measurements of fast-ion losses in the AUG tokamak. A simple model suggests that the acceleration could be explained in terms of a resonant interaction between the fast-ion orbits and the parallel electric field emerging during the ELM, when magnetic reconnection is believed to take place. The finding motivates the incorporation of a kinetic description of fast particles in ELM models and may shed light on the possible contribution of fast ions (and electrons) to the ELM stability. The impact on the overall particle and energy loss during the ELM cycle should also be investigated for a better understanding of the transient heat loads delivered to plasma-facing components and its scaling to future devices.

This work has been carried out within the framework of the EUROfusion Consortium and has received funding from the Euratom research and training program under Grant Agreement No. 633053. The views and opinions expressed herein do not necessarily reflect those of the European Commission. The support from the H2020 MarieSklodowska Curie programme (Grant No. 708257) and the Spanish Ministry of Economy and Competitiveness
(Grants No. RYC-2011-09152, No. FIS2015-69362-P, No. FJCI-201422139) is gratefully acknowledged.

*jgaldon@us.es

[1] A. G. Emslie, J. A. Miller, and J. C. Brown, Astrophys. J. 602, L69 (2004).

[2] H. S. Fu, Yu. V. Khotyaintsev, A. Vaivads, A. Retinò, and M. André, Nat. Phys. 9, 426 (2013).

[3] K. G. McClements and M. R. Turnyanskiy, Plasma Phys. Controlled Fusion 59, 014012 (2017).

[4] R. P. Lin et al., Astrophys. J. 595, L69 (2003).

[5] M. Oieroset, R. P. Lin, T. D. Phan, D. E. Larson, and S. D. Bale, Phys. Rev. Lett. 89, 195001 (2002).

[6] J. Egedal, W. Daughton, and A. Le, Nat. Phys. 8, 321 (2012).

[7] J. F. Drake, M. Swisdak, H. Che, and M. A. Shay, Nature (London) 443, 553 (2006).

[8] M. Hoshino, Phys. Rev. Lett. 108, 135003 (2012).

[9] J. T. Dahlin, J. F. Drake, and M. Swisdak, Phys. Plasmas 21, 092304 (2014).

[10] J. T. Dahlin, J. F. Drake, and M. Swisdak, Phys. Plasmas 23, 120704 (2016).

[11] H. Dreicer, Phys. Rev. 115, 238 (1959).

[12] A. Boozer, Phys. Plasmas 22, 032504 (2015).

[13] C. Paz-Soldan et al., Phys. Rev. Lett. 118, 255002 (2017).

[14] P. Helander, L. G. Eriksson, R. J. Akers, C. Byrom, C. G. Gimblett, and M. R. Tournianski, Phys. Rev. Lett. 89, 235002 (2002).

[15] S. Eilerman, J. K. Anderson, J. S. Sarff, C. B. Forest, J. A. Reusch, M. D. Nornberg, and J. Kim, Phys. Plasmas 22, 020702 (2015).

[16] S. J. Freethy, K. G. McClements, S. C. Chapman, R. O. Dendy, W. N. Lai, S. J. P. Pamela, V. F. Shevchenko, and R. G. L. Vann, Phys. Rev. Lett. 114, 125004 (2015).

[17] H. Zohm, Plasma Phys. Controlled Fusion 38, 105 (1996).

[18] A. W. Leonard, Phys. Plasmas 21, 090501 (2014).

[19] F. Wagner et al., Phys. Rev. Lett. 49, 1408 (1982).

[20] R. P. Wenninger et al., Nucl. Fusion 54, 114003 (2014).

[21] M. Garcia-Munoz, H.-U. Fahrbach, and H. Zohm, Rev. Sci. Instrum. 80, 053503 (2009).

[22] M. Salewski et al., Nucl. Fusion 52, 103008 (2012).

[23] M. Salewski et al., Nucl. Fusion 56, 106024 (2016).

[24] J. Galdon-Quiroga et al., Nucl. Fusion 58, 036005 (2018).

[25] J. Galdon-Quiroga et al., Plasma Phys. Controlled Fusion (to be published).

[26] T. Evans et al., Nat. Phys. 2, 419 (2006).

[27] W. Suttrop et al., Plasma Phys. Controlled Fusion 59, 014049 (2017).

[28] M. J. Pueschel F. Jenko, M. Schneller, T. Hauff, S. Günter, and G. Tardini, Nucl. Fusion 52, 103018 (2012).

[29] D. Pace et al., Phys. Plasmas 20, 056108 (2013).

[30] G. Huysmans and O. Czarny, Nucl. Fusion 47, 659 (2007).

[31] F. Mink, E. Wolfrum, M. Maraschek, H. Zohm, L. Horváth, F. M. Laggner, P. Manz, E. Viezzer, and U. Stroth, Plasma Phys. Controlled Fusion 58, 125013 (2016).

[32] F. Zonca, L. Chen, S. Briguglio, G. Fogaccia, G. Vlad, and X. Wang, New J. Phys. 17, 013052 (2015).

[33] L. Sanchis-Sanchez et al. (unpublished). 